Running head: AUTOMATIC EVALUATION OF ACTIONS

Evidence for the automatic evaluation of self-generated actions

Kristien Aarts, Jan De Houwer, and Gilles Pourtois

Department of Experimental-Clinical and Health Psychology, Ghent University, Belgium

Correspondence :

Gilles Pourtois

Department of Experimental-Clinical and Health Psychology

Ghent University

Henri Dunantlaan 2

9000 Gent, Belgium

Phone: +3292649144

Email: gilles.pourtois@ugent.be 


\begin{abstract}
The accuracy of simple actions is swiftly determined through specific monitoring brain systems. However, it remains unclear whether this evaluation is accompanied by a rapid and compatible emotional appraisal of the action that allows to mark incorrect actions as negative/bad and conversely correct actions as positive/good. In this study, we used a new method to decode the affective value of simple actions generated by participants during a standard Go/noGo task. Immediately after each Go/noGo action, participants responded to the valence of either a positive or a negative word. Results showed that False Alarms performed during the Go/noGo task led to a faster evaluative categorization of negative words relative to positive words. This action - word evaluative priming effect occurred when the interval between these two events was set to either 300 or $600 \mathrm{~ms}$, but not $1000 \mathrm{~ms}$. Finally, higher levels of trait anxiety were associated with a reduction of the evaluative priming effect. Our results suggest that simple actions are rapidly evaluated as positive or negative depending on the automatic monitoring of their perceived accuracy.
\end{abstract}

Keywords: action monitoring, evaluative priming, emotion, goal conduciveness, trait anxiety 


\section{Evidence for the automatic evaluation of self-generated actions}

Human beings constantly and effortlessly categorize external stimuli in their environment as good or bad. This function is adaptive because it enables us to unlock rapidly appropriate behavioral responses, for example to approach a positive stimulus or avoid a negative stimulus (Cacioppo, Priester, \& Berntson, 1993; Chen \& Bargh, 1999; Neumann, Förster, \& Strack, 2003). Evidence for automatic evaluative processing has been obtained in evaluative priming studies (De Houwer, Hermans, \& Eelen, 1998; Fazio, Sanbonmatsu, Powell, \& Kardes, 1986; Hermans, De Houwer, \& Eelen, 1994). Evaluative priming refers to the fact that reaction times (RTs) for categorizing the valence of a target word (e.g., "cold") are shorter when it is preceded by a prime with the same valence (e.g., "cancer") than when it is preceded by a prime with a different valence (e.g., "happy"). Given that there is very little time between the onset of the prime and the onset of the target (typically less than $300 \mathrm{~ms}$ ) and participants are asked to ignore the primes, these results suggest that the valence of the prime is processed automatically in the sense of rapidly and unintentionally. Evaluative priming has already been observed for a wide range of external stimuli in the environment, including words (Fazio et al., 1986), pictures (Hermans et al., 1994), black and white line drawings (Giner-Sorolla, Garcia, \& Bargh, 1999), motivationally-relevant stimuli (i.e., rewarded and unrewarded colors; (Moors \& De Houwer, 2001), odors (Hermans, Baeyens, \& Eelen, 1998) and tones (Reber, Haerter, \& Sollberger, 1999).

Presumably, automatic evaluation is a generic function and does not only concern external stimuli in the environment, but also self-generated actions. Actions in response to stimuli are usually deemed conducive or obstructive depending on their actual match with goals stored in long term memory (Scherer, 1984, 1988). Indirect evidence supporting this view comes from recent psychophysiology studies showing that unwanted response errors (i.e., goal obstructive events) yield larger skin conductance responses and greater heart rate deceleration than 
correct decisions (Hajcak, McDonald, \& Simons, 2003b), as well as a larger startle potentiation (Hajcak \& Foti, 2008) and differential early activation in the amygdala (Pourtois et al., 2010). These results suggest that errors may be perceived as aversive events, and accordingly be associated with enhanced arousal within the autonomic nervous system. It has also been shown that through conditioning, a specific action (e.g., a key press) can become aversive as evidenced by the fact that the selection of the action is faster by the presence of an irrelevant negative word (Beckers, De Houwer, \& Eelen, 2002). Although these studies give first hints on the acquired emotional value of specific actions, they do not inform us about whether valence specific effects can be obtained as a function of the perceived goal conduciveness of simple self-generated actions. Earlier studies showed that physiological measures such as startle potentiation reflect not only valence but also arousal (Vansteenwegen, Crombez, Baeyens, \& Eelen, 1998). Moreover, it is likely that the posterror detection changes in autonomic or brain activity that were observed in previous studies merely reflect enhanced arousal (Hajcak \& Foti, 2008) or attention orienting (Notebaert et al., 2009) rather than a genuine affective marking of these actions as negative events. Accordingly, it remains to be shown more directly whether response errors and correct responses are processed "online" along a genuine evaluative dimension, eventually allowing a negative value to be mapped onto errors and conversely a positive value onto correct responses. Evaluative priming is generally regarded as a task that allows one to capture online evaluative reactions (e.g., De Houwer, Teige-Mocigemba, Spruyt, \& Moors, 2010; Fazio, 2001) and thus could provide unique information about this issue.

Earlier event-related potential (ERP) studies have also provided evidence for an early emotional processing of errors following their onset (Luu, Collins, \& Tucker, 2000), as well as for an early differential appraisal of errors compared to correct hits (Aarts \& Pourtois, 2010; Hajcak, McDonald, \& Simons, 2004). Moreover, it has been found that early brain 
responses related to error detection are typically enhanced in high trait anxious individuals. This neurological evidence is, however, also limited in that the observed neurophysiological effects were most often not accompanied by detectable affect-related changes in the behavior in these earlier studies (Hajcak \& Foti, 2008; Hajcak, McDonald, \& Simons, 2003a; Hajcak et al., 2004). Hence, it is unclear whether overactive early error-related brain activities (including the ERN) seen in high anxious participants reflect a general impairment in cognitive control or action monitoring, or alternatively, a selective difficulty in ascribing "online" a negative value to response errors. Furthermore, these earlier studies focussed mainly on response errors. Hence, much less is known about a possible symmetric affective tagging of correct actions as positive events by generic internal action monitoring brain systems. In other words, it still needs to be determined at the behavioral level whether incorrect actions are automatically categorized as negative events relative to correct actions, while conversely correct actions would implicitly be associated with positive emotions, relative to response errors.

We addressed this question using a novel experimental paradigm suited to decode online the emotional value of simple self-generated actions performed during a standard Go/noGo task by healthy adult participants. Participants performed a speeded Go/noGo task (Vocat, Pourtois, \& Vuilleumier, 2008), which was combined with an evaluative word categorization task. Unbeknown to participants, actions performed during the Go/noGo task (either correct or incorrect responses) served as primes whereas the words (positive or negative) were used as targets. In line with the logic underlying evaluative priming effects, we predicted that the time needed to categorize a target word would be systematically influenced by the putative valence of the preceding action, the latter being presumably decoded rapidly following or even during action execution in specific cognitive and emotion control systems (De Bruijn, de Lange, von Cramon, \& Ullsperger, 2009). More precisely, we expected participants to be 
faster to categorize a target word as negative if the preceding action was incorrect and to categorize a target word as positive if the preceding action was correct. Our prediction was based on the idea that self-generated actions are appraised quickly as being either goal conducive or goal obstructive events. Appraisal theories of emotion imply that, in the former case, a positive value or valence is generated, while in the latter case, a negative valence is temporarily activated (Scherer, 1988). In this framework, errors automatically evoke a negative reaction because they mismatch with the goals set out by the task.

Figure 1.

A

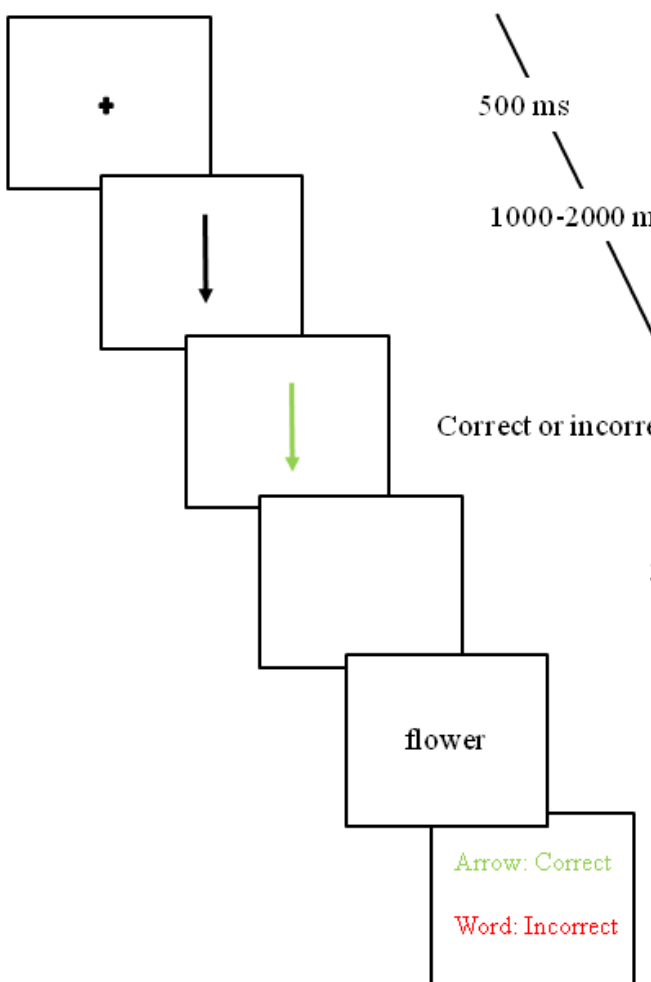

B

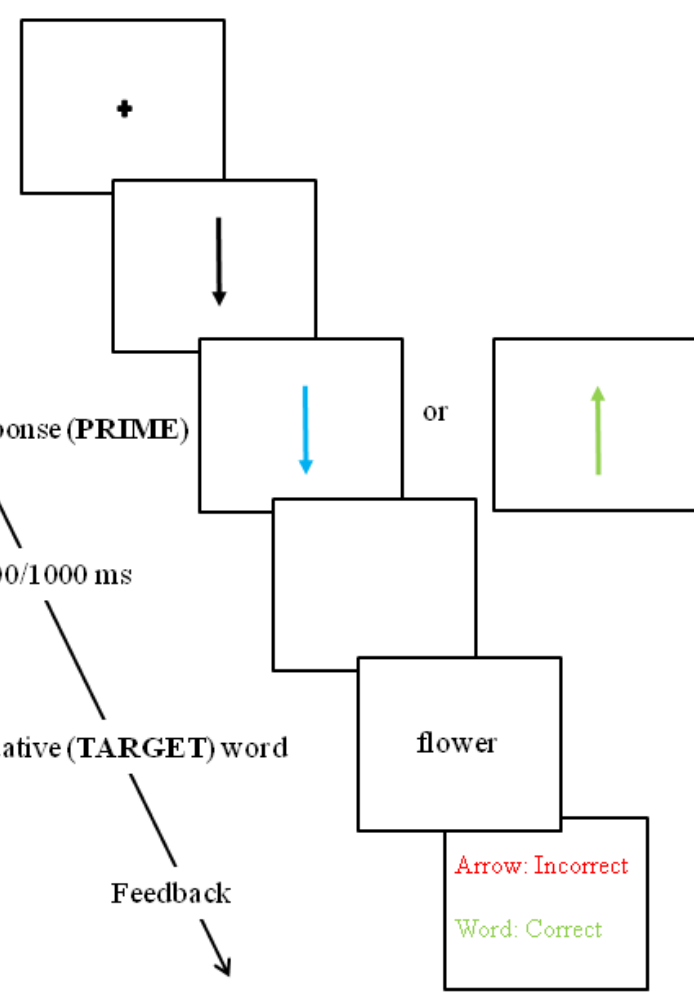

We also examined some of the functional properties of automatic evaluation of correct and incorrect actions. More specifically, we tested whether the effect was moderated by the time between the action and the target word and by the affective disposition of the participants. Previous studies with word primes and word targets reported reliable evaluative priming effects with a short stimulus onset asynchronies (SOAs) between prime and target (i.e., 300 
ms or less) but not with long SOAs (e.g., 1000 ms; see Fazio et al., 1986; De Houwer et al., 1998; Hermans et al., 1994). Based on these results, it was concluded that the automatic evaluation of words is a fast acting automatic process. In our first two experiments, the length of the SOA between the self-generated action and the presentation of the target word was constant and set to $300 \mathrm{~ms}$. It was increased to $600 \mathrm{~ms}$ in Experiment 3 and to $1000 \mathrm{~ms}$ in Experiment 4. If the evaluation of correct and incorrect actions is also a fast acting automatic process (see Pourtois et al., 2010, for converging neuroscientific evidence) then evaluative priming should be observed at short (300 ms) but not long (1000 ms) SOAs.

Because high anxious individuals are typically more sensitive and reactive to negative information (including errors), one might predict a stronger association between the (online) monitoring of response errors and negative emotion words in these individuals. However, in a recent ERP study, Aarts and Pourtois (2012) observed that high anxious individuals exhibited a selective deficit in relating the valence of external evaluative feedback (positive or negative) to the value of their self-generated actions (correct or incorrect), as shown by amplitude changes at the level of the FRN component. More specifically, high anxious participants did not show a normal amplitude variation of the FRN (time-locked to the evaluative feedback) as a function of the perceived "correctness" of their actions (Aarts \& Pourtois, 2012). Therefore, we predicted that trait anxiety might actually decrease the size of the evaluative priming effect, bearing in mind that this effect is assumed to depend upon an automatic and fast affective marking of self-generated actions (responses errors/bad vs. correct responses/good) and this specific process that may be impaired in high anxious participants (Aarts \& Pourtois, 2010).

\section{Method}

\section{Participants}


Twenty-one first-year female undergraduate psychology students (Age: $M=18.52 ;$ SEM $=$ $0.40 ;$ Range $=17-25)$ participated in Experiment 1 . Fifteen undergraduate students (14 women; Age: $M=21.4, S E M=.38$, Range $=18-23$ ) took part in Experiment 2. Twenty-two undergraduate students participated in Experiment 3 (19 women; Age: $M=21.73 ;$ SEM $=.50$, Range $=19$ - 28). Finally, 20 undergraduate students took part in Experiment 4 (18 women; Age: $M=23.05, S E M=.86$, Range $=18-26$ ). All participants were right-handed, native Dutch speakers who did not have a history of neurological or psychiatric disease and had normal or corrected-to-normal vision. The study was approved by the local ethics committee. All students participated in exchange for course credits or for money (10 Euro).

\section{Stimuli}

In the Go/noGo task, visual stimuli consisted of an arrow (subtending $11.4 \circ \times 0.05^{\circ}$ of visual angle at a $60 \mathrm{~cm}$ viewing distance), that was presented in the center of a white homogenous background, and oriented either upward or downward (see Figure 1). The arrow was first black, and could then turn either green or turquoise. These two colors were matched for luminance. These different combinations of color and orientation were used as cues in the Go/noGo task.

In the evaluative categorization task, targets were 30 positive and 30 negative words, either nouns or adjectives (see Table 1), and were selected from the Dutch affective rating list of Hermans and De Houwer (1994). T-tests showed that these positive and negative words differed significantly on the affective dimension, $t(58)=36.57, p<.001, \eta_{p}{ }^{2}=.95$, but not on the familiarity dimension, $t<1$, nor with respect to the number of letters, $t<1$. Because actions functioned as primes, we also made sure that the list of positive target words did not contain more action-related words than the list of negative target words (one and two actionrelated words, respectively). 
Table 1.

Target words selected from the Dutch affective rating list of Hermans and De Houwer (1994)

\begin{tabular}{|c|c|c|c|}
\hline \multicolumn{2}{|c|}{ Positive targets } & \multicolumn{2}{|c|}{ Negative targets } \\
\hline Hawaii (Hawaii) & trouw (fidelity) & ruw (rude) & stank (stench) \\
\hline engel (angel) & lente (spring) & haat (hate) & drugs (drugs) \\
\hline goud (gold) & baby (baby) & moord (murder) & virus (virus) \\
\hline regenboog (rainbow) & parfum (parfume) & aids (aids) & puist (pustule) \\
\hline bruid (bride) & knuffel (hug) & vals (false) & zweer (sore) \\
\hline applaus (applause) & feest (part) & pijn (pain) & oorlog (war) \\
\hline hemel (heaven) & oprecht (sincere) & dief (thief) & kanker (cancer) \\
\hline geboorte (birth) & zomer (summer) & dood (dead) & hitler (hitler) \\
\hline vrede (peace) & humor (humor) & graf (tomb) & geweren (guns) \\
\hline spel (game) & bloemen (flowers) & sluw (sly) & ongeval (accident) \\
\hline geschenk (gift) & omhelzing (embrace) & hoer (hore) & brutaal (impudent) \\
\hline cadeau (present) & vakantie (holiday) & koud (cold) & vulgair (vulgar) \\
\hline trots (proud) & droom (dream) & zwak (weak) & ongezond (unhealthy) \\
\hline melodie (melody) & leven (life) & spin (spider) & hatelijk (hasty) \\
\hline romantiek (romanticism) & liefde (love) & vuil (dirty) & vijandig (hostile) \\
\hline
\end{tabular}

\section{Procedure}

Participants performed a standard speeded Go/noGo task (Vocat et al., 2008) interleaved with a visual word categorization task (see Figure 1). Actions performed during the speeded Go/noGo task actually served as primes whereas words were deemed targets in analogy with a conventional prime-target sequence during evaluative priming. After a practice block 
including 24 trials, the experiment was divided into 3 main sessions, each starting with a training block (containing 28 trials: 20 Go and 8 noGo trials), followed by two test blocks (each containing 72 trials: 48 Go and 24 noGo trials). Note that participants were unaware that training blocks were actually used as calibration blocks to compute a RT limit (see further) in order to evaluate speed during the two following test blocks. Trial presentation was randomized within blocks. Between blocks, a small break (no longer than 5 min) was introduced. The whole experiment included 540 trials and lasted on average $50 \mathrm{~min}$. Stimulus presentation and response recording were controlled using E-prime software (V2.0., http://www.pstnet.com/products/e-prime/).

In this task, each trial started with a fixation cross that lasted for $500 \mathrm{~ms}$. Afterwards, a black arrow, either oriented up or down, was presented at the position previously occupied by the fixation cross. After a variable interval ranging from $1000 \mathrm{~ms}$ to $2000 \mathrm{~ms}$, the black arrow became either green or turquoise while its orientation could either remain identical or shift in the opposite direction compared to the initial black arrow. When the black arrow turned green and the orientation remained unchanged, participants were instructed to press a pre-defined button of the response box as fast as possible with the index finger of their left hand (Go trials $-66 \%$ of all trials). However, participants had to withhold responding when either the arrow became green but changed orientation, or when the arrow became turquoise and kept its initial orientation, enabling two noGo trial types (33\% of all trials). Instructions emphasized both speed and accuracy, such that not only accuracy, but also the speed of the responses was later evaluated as being correct or incorrect.

For each trial, speed was evaluated using an individually calibrated RT limit computed during a training block that preceded each session of two test blocks. This limit was thus calculated and updated three times in total (before Blocks 1 and 2 - Session 1, before Blocks 3 and $4-$ Session 2, and before Blocks 5 and 6 - Session 3). This allowed us to deal with 
unspecific learning effects over time and maintain a similar number of correct and incorrect responses throughout the experiment. For the first session, the upper limit was set to $70 \%$ of the mean RT from the first training block. For the two subsequent sessions, this upper limit was updated and set to $80 \%$ of the mean RT during the respective training block. Hence, this procedure required participants to respond at least 30\% faster (first session) or $20 \%$ faster (second and third sessions) on Go trials than their average speed during the respective training block. This procedure ensured a sufficient number of response errors on noGo trials and allowed us to distinguish between Fast Hits (i.e., responses on Go trials that were emitted more quickly than the individually-titrated RT limit) or Slow Hits (i.e., responses on Go trials that took longer than the RT limit). Errors were formally defined as overt responses on noGo trials (i.e., FAs), while correct inhibitions corresponded to withheld responses on the same noGo trials.

Three hundred milliseconds after an action was executed, a target word was presented. The same 300 ms SOA was used in Experiment 2 because this experiment was mainly run to provide a replication of the results obtained in Experiment 1. The SOA was set to $600 \mathrm{~ms}$ in Experiment 3 and to $1000 \mathrm{~ms}$ in Experiment 4 in order to assess whether an evaluative priming effect was sensitive to the time elapsed between prime (action) and target (word). For correct inhibitions, the target word was presented $1500 \mathrm{~ms}$ after the presentation of the colored arrow plus the length of the SOA. Participants were instructed to categorize the valence of the target word (positive or negative) as fast and as accurately as possible by pressing one of two predefined keys of the response box using their dominant hand. Hence, the evaluative word categorization task was executed with a different effect or than the Go/noGo task. The target word remained on the screen until the participant responded or $3000 \mathrm{~ms}$ elapsed. In order to balance the presentation of positive vs. negative words following Fast Hits, Slow Hits, Correct Inhibitions, and FAs, the target word that was 
presented following an action was selected randomly on each trial. After the word categorization, participants received feedback informing them about their accuracy for the two consecutive tasks. A feedback screen was given to participants after each individual trial. It included general feedback (accuracy and speed) on the go/nogo task (top of the screen) and feedback (accuracy) on the subsequent emotion word task categorization task (bottom of the screen) (see Figure 1). The feedback for the Go/noGo task indicated whether the performed action was correct (and fast enough), incorrect, or too slow, while the feedback for the word categorization could be either that the response was correct or incorrect. Both feedback signals remained on the screen for $2000 \mathrm{~ms}$.

Accuracy and RTs (correct responses) for the evaluative word categorization task were analyzed using repeated measures analyses of variance (ANOVAs) as a function of (i) the valence of the target word (either positive or negative) and (ii) the type of action (FA, Fast Hit or Slow Hit) preceding word presentation. We did not include in these analyses RTs and errors for target categorization when a response on noGo trials was correctly inhibited because no overt (Go) action was performed in this condition. Note that on noGo trials, the interval between the start of the presentation of the noGo stimulus and start of the presentations of the target word was at least $1800 \mathrm{~ms}(1500 \mathrm{~ms}$ presentation of the noGo stimulus plus the length of the corresponding SOA; $300 \mathrm{~ms}, 600 \mathrm{~ms}$ or $1000 \mathrm{~ms}$ ). Given that evaluative priming effects are typically observed only at short SOAs (i.e., less than $1000 \mathrm{~ms}$; e.g., Fazio et al., 1986), we did not expect to find a significant evaluative priming effect following the correct rejections. Additional statistical analyses confirmed that the evaluative categorization was not influenced by these preceding correct inhibitions as the speed to categorize negative words did not differ significantly from the speed to categorize positive words (all $T \mathrm{~s}<1$ in Experiments 1-4). 
After completion of the three experimental sessions, the Dutch version of the trait version of the Spielberger State-Trait Anxiety Inventory (STAI) (Defares, van der Ploeg, \& Spielberger, 1979; Spielberger, 1983) was filled out by the participants.

\section{Results}

In all four experiments, trials with RTs shorter than $150 \mathrm{~ms}$ or longer than $500 \mathrm{~ms}$ in the Go/noGo task were discarded, as were trials in which the RT on the evaluative categorization task exceeded 2.5 SD from the mean RT per condition (see Table 2). Two participants (female) were not included in the statistical analyses of the data of Experiment 4 because they did not commit sufficient (i.e., minimum 10) FAs to compute reliable accuracy or RT estimates for each condition separately (i.e., positive words following FAs vs. negative words following FAs). Hence, in Experiment 4, only the data of the remaining 18 participants were included in the analysis.

\begin{tabular}{|c|c|c|c|c|c|c|c|c|c|}
\hline \multicolumn{10}{|c|}{$\begin{array}{l}\text { Percentages outlier trials in the Go/noGo task (< } \\
\text { categorization task (< or > than } R T s \pm 2.5 S D)\end{array}$} \\
\hline \multirow[b]{2}{*}{ Criterium } & \multirow[b]{2}{*}{ Condition } & \multicolumn{2}{|c|}{ Exp 1} & \multicolumn{2}{|c|}{$\operatorname{Exp} 2$} & \multicolumn{2}{|c|}{ Exp 3} & \multicolumn{2}{|c|}{$\operatorname{Exp} 4$} \\
\hline & & $M$ & SEM & $M$ & SEM & $M$ & SEM & $M$ & SEM \\
\hline \multirow[t]{2}{*}{$<150 \mathrm{~ms}$} & FAs & 4.25 & 1.27 & 3.02 & 0.87 & 5.78 & 1.33 & 6.63 & 2.21 \\
\hline & Fast Hits & 6.69 & 2.02 & 4.47 & 1.76 & 9.34 & 2.40 & 10.44 & 4.33 \\
\hline \multirow[t]{2}{*}{$>500 \mathrm{~ms}$} & FAs & 3.04 & 1.71 & 0.90 & 0.32 & 2.37 & 1.36 & 2.81 & 0.97 \\
\hline & Slow Hits & 6.45 & 1.11 & 3.73 & 1.23 & 7.90 & 1.92 & 7.16 & 1.08 \\
\hline$>$ or <than & Negative & 2.89 & 0.21 & 2.91 & 0.22 & 5.60 & 1.13 & 3.30 & 0.21 \\
\hline $\mathrm{RTs} \pm 2.5 \mathrm{SD}$ & Positive & 2.82 & 0.15 & 3.10 & 0.28 & 3.30 & 0.76 & 3.38 & 0.19 \\
\hline
\end{tabular}




\section{Evaluative Categorization Task}

\section{Speed.}

We first performed an omnibus ANOVA on correct RTs (i.e. correct word categorization responses) collected across the four experiments to verify that the categorization of the target word was reliably influenced by the putative affective value of the preceding action, only when a short (Experiments 1-3) but not long SOA (Experiment 4) was used between these two events. This analysis confirmed a significant three-way interaction between action type (FA, Fast Hit or Slow Hit), word type (positive or negative) and SOA (300, 600 or 1000 ms), $F(4,146)=3.96, p<.01, \eta_{p}{ }^{2}=.10$. In a second step, we performed an ANOVA on the mean RTs for correct word categorization responses, for each experiment separately.

In Experiment 1, the ANOVA revealed a significant interaction effect between action type (FAs, slow Hits, fast Hits) and word type (positive or negative), $F(2,40)=13.51, p<.001$, $\eta_{p}{ }^{2}=.40$. This interaction resulted from faster evaluative categorizations when the valence of the word was congruent with the putative affective value of the action. More specifically, RTs for negative words following FAs were shorter compared to RTs for positive words following FAs, $t(20)=-2.57, p<.05, \eta_{p}{ }^{2}=.25$, while symmetrically, participants tended to categorize positive words faster compared to negative words when they followed Fast Hits, $t(20)=1.81, p=.08, \eta_{p}{ }^{2}=.14$. Following Slow Hits, no significant RT difference emerged between negative and positive words, $t<1$. The main effect of word type was not significant, $F<1$. By contrast, the ANOVA revealed a significant main effect of action type, $F(2,40)=$ 26.04, $p<.001, \eta_{p}{ }^{2}=.57$, reflecting longer RTs for words following FAs compared to words following either Fast Hits, $F(1,20)=30.00, p<.001, \eta_{p}{ }^{2}=.60$, or Slow Hits, $F(1,20)=$ $30.25, p<.001, \eta_{p}{ }^{2}=.60$, an effect in line with a systematic post-error slowing (Danielmeier \& Ullsperger, 2011; Rabbitt, 1966). Because this general RT slowing following FAs compared to Fast Hits might lead to an artificial increase in evaluative priming for FAs 
compared to Fast Hits, we also analyzed log transformed RTs. This analysis confirmed a significant interaction effect between action type and word type, $F(2,40)=16.41, p<.001$, $\eta_{p}{ }^{2}=.45$. RTs for negative words following FAs were shorter compared to RTs for positive words following FAs, $t(20)=-2.23, p=.04, \eta_{p}{ }^{2}=.20$, while participants categorized positive words faster compared to negative words when they followed Fast Hits, $t(20)=3.71, p<$ $.001, \eta_{p}{ }^{2}=.41$. Shorter RTs were also observed for words following Fast Hits compared to Slow Hits, $F(1,20)=6.10, p<.05, \eta_{p}{ }^{2}=.23($ see Figure $2 \mathrm{~A})$.

An identical interaction effect between action type and word type was found in Experiment $2, F(1,28)=13.60, p<.001, \eta_{p}{ }^{2}=.49$ (negative vs. positive words: FAs: $t(14)=$ $-3.28, p<.01$; Fast Hits: $\left.t(14)=-2.51, p<.05, \eta_{p}{ }^{2}=.43\right)$, and Experiment $3, F(2,42)=$ 14.62, $p<.001, \eta_{p}{ }^{2}=.41$ (negative vs. positive words: FAs: $t(21)=-8.74, p<.01, \eta_{p}{ }^{2}=.29$; Fast Hits: $\left.t(14)=12.31, p<.005, \eta_{p}{ }^{2}=.37\right)$, but not in Experiment $4, F<1$. Also no effect of word type was observed for Experiments 2-4 (Experiment 2: $F(1,28)=2.02, p>.10, \eta_{p}{ }^{2}=$ .13 ; Experiment 3: $F(1,42)=1.56, \mathrm{p}>.10, \eta_{p}{ }^{2}=.07$; Experiment $4: F<1, \eta_{p}{ }^{2}=.02$ ) while the post-error slowing effect was observed in all experiments (Experiment 2: $F(2,40)=$ 26.80, $p<.001, \eta_{p}{ }^{2}=.66$; Experiment 3: $F(2,42)=5.13, p<.05, \eta_{p}{ }^{2}=.20$; Experiment 4: $F(2,34)=15.49, p<.001, \eta_{p}^{2}=.48$; see Figure 2BCD). 

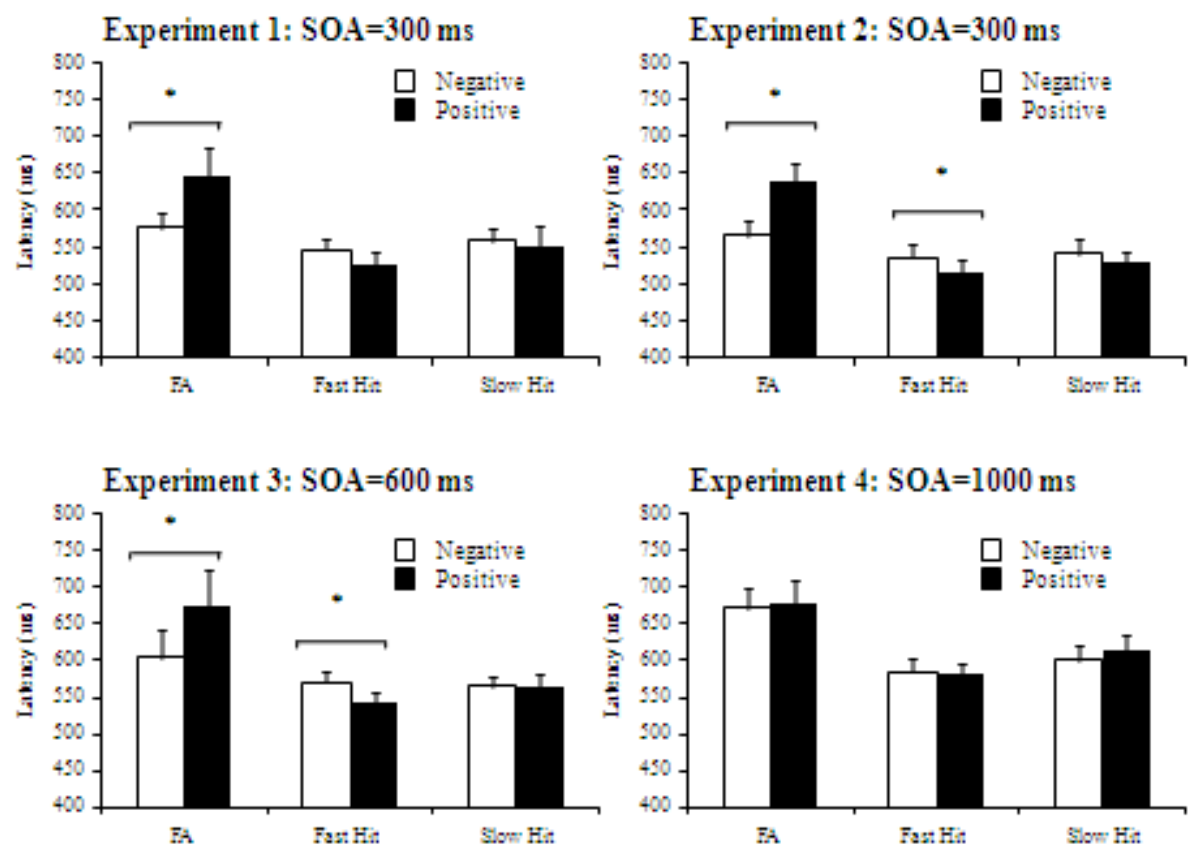

Figure 2.

\section{Accuracy.}

We first performed an omnibus ANOVA on the accuracy data collected across the four experiments. This analysis showed a significant three-way interaction between action type (3 levels), word type (2 levels) and SOA (3 levels), $F(4,146)=2.91, p<.05, \eta_{p}{ }^{2}=.07$. In a second step, we performed an ANOVA on the percentage correct word categorization responses, for each experiment separately.

In Experiment 1, the ANOVA performed on accuracy data (i.e., \% correct responses) revealed a significant interaction between action type (FA, Fast Hit, Slow Hit) and word type (positive, negative), $F(2,40)=6.05 . p<.01, \eta_{p}{ }^{2}=.23$. This interaction indicated that participants were less accurate to categorize positive compared to negative words following FAs, $t(20)=2.81, p<.05, \eta_{p}{ }^{2}=.28$. Accuracy was similar for categorizing positive and negative words following either Fast Hits, $t(20)=-1.38, p>.10, \eta_{p}{ }^{2}=.09$, or Slow Hits, $t(20)$ $=-1.01, p>.10, \eta_{p}^{2}=.05$. Furthermore, the main effect of action type approached 
significance, $F(2,40)=3.00, p=.06, \eta_{p}{ }^{2}=.13$, indicating higher accuracy following Fast Hits compared to FAs, $F(1,40)=7.58, p<.05, \eta_{p}{ }^{2}=.28$ (see Table 3). Finally, the main effect of word type was not significant, $F<1$.

A similar interaction between action type and word type was observed in Experiment 2, $F(1,28)=14.39, p<.001, \eta_{p}{ }^{2}=.51$, and Experiment $3, F(2,42)=14.62, p<.001, \eta_{p}{ }^{2}=.41$, but not in Experiment 4, $F(1,34)=1.63, p>.10, \eta_{p}{ }^{2}=.09$. Also, a similar effect of action type was observed in Experiments 2-4 (Experiment 2: $F(2,28)=4.17, p<.05, \eta_{p}{ }^{2}=.23$; Experiment $3, F(2,42)=9.28, p<.001, \eta_{p}{ }^{2}=.30$; Experiment $4, F(2,34)=8.17, p=.001$, $\left.\eta_{p}{ }^{2}=.33\right)$. A significant effect of word type was observed in Experiment $2, F(1,28)=6.11, p$ $<.05, \eta_{p}{ }^{2}=.30$ and Experiment $3, F(1,42)=6.56, p<.05, \eta_{p}{ }^{2}=.24$, with less accurate categorizing for negative compared to positive words. This effect was not observed in Experiment $4, F(1,34)=1.63, p>.10, \eta_{p}^{2}=.09($ see Figure 3$)$.
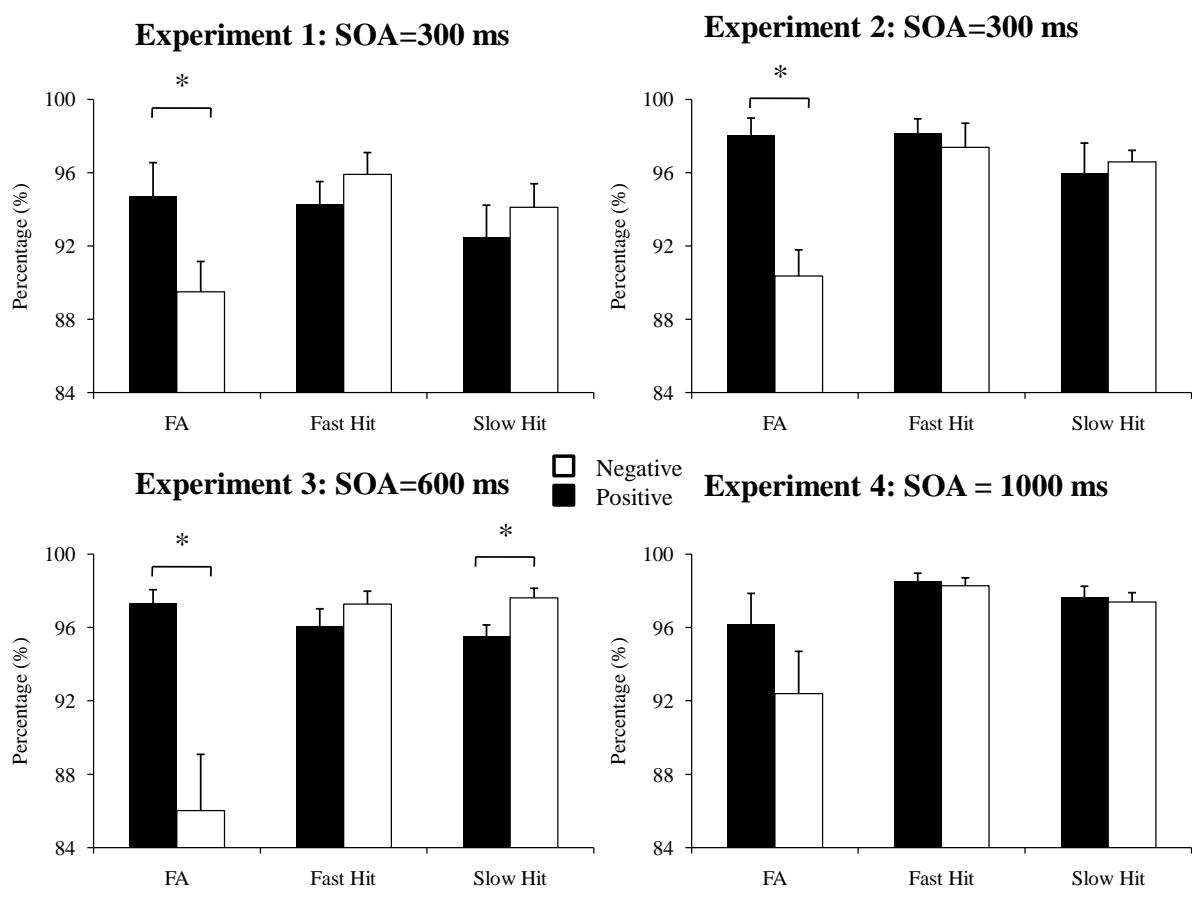

Experiment 4: SOA $=1000 \mathrm{~ms}$

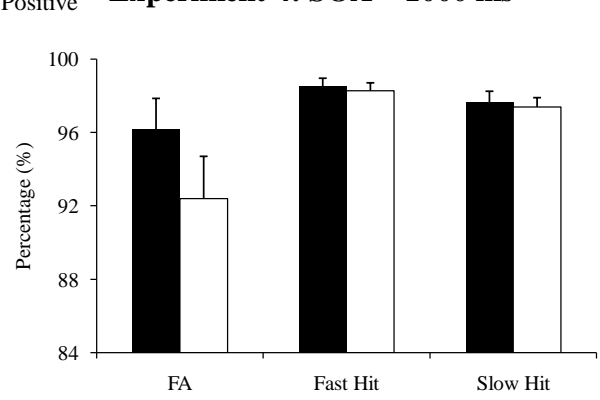

Figure 3.

\section{Go/noGO task}


We determined the percentage of action types (FA, Fast Hit, Slow Hit) made during the Go/noGo task, as well as the speed with which these responses were made. The analysis performed on the percentage of action types revealed a significant main effect of action type, $F(2,40)=12.22, p<.001, \eta_{p}^{2}=.38$. The percentage of FAs was similar to the percentage of Fast Hits, $t<1$. However, participants made significantly less Fast Hits than Slow Hits, $t(20)$ $=-4.61, p<.001, \eta_{p}{ }^{2}=.52$. As expected, a significant main effect of action type was also observed for speed, $F(2,40)=275.36, p<.001, \eta_{p}{ }^{2}=.93$. RTs for Slow Hits were longer than RTs for Fast Hits, $t(20)=-24.37, p<.001, \eta_{p}{ }^{2}=.97$, while RTs for FAs were shorter than RTs for Slow Hits, $t(20)=3.14, p=.005, \eta_{p}{ }^{2}=.94$, but longer than RTs for Fast Hits, $t(20)=-17.38, p<.001, \eta_{p}^{2}=.33$ (see Table 4). A similar main effect of action type on the percentage of actions and speed was also observed in Experiments 2-4 (see Table 3).

Table 3.

Mean percentage (\%), latencies (ms) and effect sizes related to the type of action during the Go/noGo task.

\begin{tabular}{|c|c|c|c|c|c|c|}
\hline & & & $\operatorname{Exp} 1$ & $\operatorname{Exp} 2$ & $\operatorname{Exp} 3$ & $\operatorname{Exp} 4$ \\
\hline \multirow[t]{10}{*}{ Percentage } & FAs & $\mathrm{M}$ & 40.34 & 41.99 & 42.86 & 31.87 \\
\hline & & SEM & 4.39 & 3.98 & 4.24 & 3.61 \\
\hline & Fast Hits & M & 39.42 & 28.55 & 38.67 & 37.18 \\
\hline & & SEM & 2.29 & 3.87 & 3.11 & 3.38 \\
\hline & Slow Hits & M & 60.57 & 71.45 & 61.33 & 62.82 \\
\hline & & SEM & 2.29 & 3.87 & 3.11 & 3.38 \\
\hline & Effect of action & $\eta_{p}^{2}$ & $.38 * * *$ & $.63 * * *$ & $.31 * * *$ & $.51 * * *$ \\
\hline & FA vs. Fast & $\eta_{p}^{2}$ & .00 & $.29 *$ & .02 & .06 \\
\hline & FA vs. Slow & $\eta_{p}^{2}$ & $.43 * * *$ & $.68 * * *$ & $.54 * * *$ & $.72 * * *$ \\
\hline & Fast vs. Slow & $\eta_{p}^{2}$ & $.52 * * *$ & $.69 * * *$ & $.39 * *$ & $.46^{* *}$ \\
\hline
\end{tabular}




\begin{tabular}{|c|c|c|c|c|c|c|}
\hline \multirow{2}{*}{\multicolumn{2}{|c|}{ Speed }} & M & 234.7 & 222.40 & 223.78 & 229.00 \\
\hline & & SEM & 5.33 & 2.66 & 4.62 & 5.69 \\
\hline & \multirow[t]{2}{*}{ Fast Hits } & M & 221.77 & 204.93 & 209.25 & 223.50 \\
\hline & & SEM & 6.34 & 5.76 & 7.23 & 8.32 \\
\hline & \multirow[t]{2}{*}{ Slow Hits } & M & 307.50 & 276.60 & 286.51 & 301.61 \\
\hline & & SEM & 7.02 & 3.80 & 8.32 & 8.12 \\
\hline & Effect of action & $\eta_{p}{ }^{2}$ & $.93 * * *$ & $.91 * * *$ & $.60 * * *$ & $.88 * * *$ \\
\hline & FA vs. Fast & $\eta_{p}^{2}$ & $.33 * * *$ & $.44 * *$ & $.36 * *$ & .06 \\
\hline & FA vs. Slow & $\eta_{p}^{2}$ & $.94 * *$ & $.96 * * *$ & $.85 * * *$ & $.90 * * *$ \\
\hline
\end{tabular}

Note. ${ }^{*} p<.05, * * p<.01, * * * p<.001$

\section{Relation between trait anxiety levels and the magnitude of the evaluative priming effect}

To put to the test our third prediction, we assessed whether levels of trait anxiety of our participants (STAI-T all: $M=36.93 ; S E M=1.50$; median split - STAI-T low: $M=28.23$; $S E M=.90 ;$ STAI-T high: $M=44.00 ; S E M=1.84)$ were related to the size of the evaluative priming effect. To address this, for each participant of Experiments 1-3 (i.e., all experiments in which a significant evaluative priming effect was found), the magnitude of evaluative priming was calculated as the difference in RT between incongruent action-word pairs (i.e., FA-positive and Fast Hit-negative) and congruent action-word pairs (i.e., FA-negative and Fast Hit-positive). The larger this difference score, the higher the influence of the preceding affective value of the action on the current evaluative categorization. Using a standard Pearson coefficient correlation analysis, we found across participants of Experiments 1-3 a significant negative correlation between levels of trait anxiety and these evaluative priming scores, $, r=-.28, p<.05$ (see Figure 4). This correlation showed that participants with higher levels of trait anxiety had a smaller evaluative priming effect. When including the non- 
significant evaluative priming results of Experiment 4 in this analysis, the correlation was no longer significant, $r=-.19, p>.10$.

Figure 4.

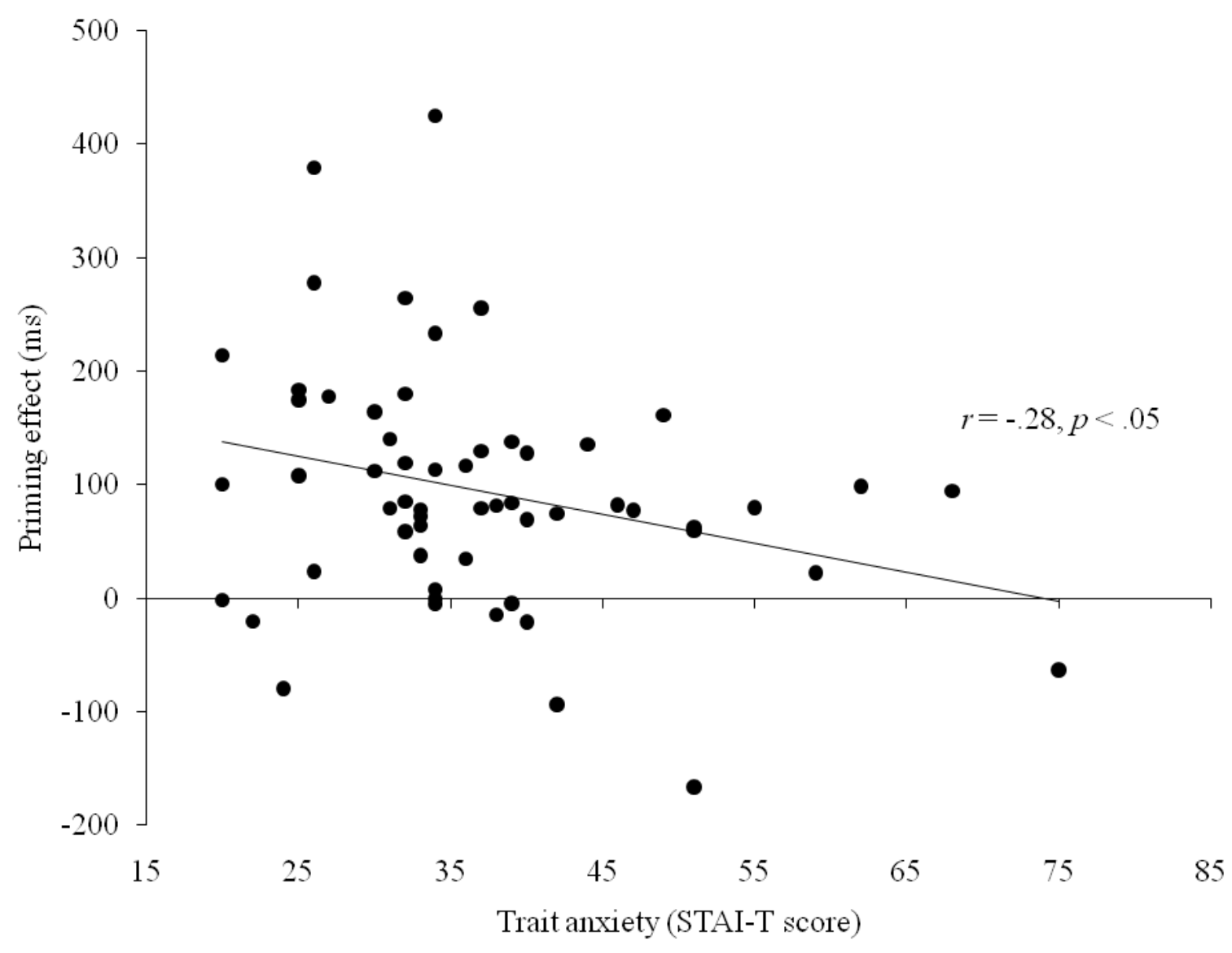

\section{Discussion}

The results of our experiments reveal that simple self-generated actions during a speeded Go/noGo task are swiftly evaluated along a negative-positive dimension. This internal appraisal influences the valence categorization of an immediately following target word, even though these two different and non-overlapping events (i.e., action and word) belong to two 
clearly separated tasks performed with two different effectors. Our findings have several important implications that we address below.

\section{Affective value of the action primes evaluative categorization}

We are the first to report evaluative priming effects that are triggered by the putative affective value which is rapidly and in an online manner assigned to self-generated actions (correct vs. incorrect) via an internal meta cognitive feedback mechanism (Fernandez-Duque, Baird, \& Posner, 2000; Winkielman, Schwarz, Fazendeiro, \& Reber, 2003). These evaluative priming effects suggest that FAs were evaluated as more negative compared to Hits (either Fast or Slow, see results of Experiments 1-3) while Fast Hits were evaluated as more positive compared to FAs (see results of Experiments 2-3). Therefore, the added value of our evaluative priming method is that it enables to access a fast valence tagging process that operates during action monitoring, as opposed to reactive arousal or attention effects following error detection for example. As such, our behavioral results go beyond earlier studies showing that different psychophysiological reactions, like larger skin conductance responses, greater heart rate deceleration and larger startle potentiation usually follow incorrect compared to correct actions (Hajcak \& Foti, 2008; Hajcak et al., 2003b). The novel contribution of our study is to show that beyond these enhanced arousal or attention orienting effects following the detection of these adverse events, dedicated internal monitoring systems enable organisms to rapidly map specific affective values (either negative or positive) onto self-generated actions (either incorrect or correct). Indirect evidence for this idea was already provided by earlier ERP studies showing that the rapid processing and monitoring of response errors involved an emotional component that might be altered in (trait) anxiety or negative affect (Aarts \& Pourtois, 2010; Hajcak et al., 2004). The results of the present study add to the literature that this mechanism appears to operate along a genuine valence dimension, which is not restricted to errors or a specific class of deviant outcomes (De Bruijn 
et al., 2009). Hence, ERP measurements used in combination with the present evaluative priming experimental design might stimulate future studies exploring the functional meaning of early error-related ERP components (including the ERN and Pe). In addition to showing that actions performed during a simple Go/noGo task are actually quickly evaluated as negative or positive, our results suggest that this affective marking of the action functions at an abstract level of action representation, as opposed to being bound to a specific motor output or command. The latter conclusion is supported by the fact that the exact same key presses were performed for correct (either Fast or Slow Hits) and incorrect actions (FAs) during our Go/noGo task. This abstract online affective appraisal of the action may in fact concern goal conduciveness (Frijda, 1987; Scherer, 1984, 1988), that is, an evaluation of whether an action is conducive (positive/Fast Hits) or obstructive (negative/FAs) for reaching the goals set out by the Go/NoGo task.

We hypothesize that the affective value of an action is determined primarily based on its perceived goal conduciveness. A response error is a goal obstructive event, while a correct response (fast hit) is deemed goal conducive. Appraisal theories of emotion (Scherer, 1988) postulate that these goal-obstructive events have the propensity to acquire a negative connotation, and symmetrically goal conducive events acquire a positive valence. Further studies are needed to confirm that these specific appraisal checks are causally related to the expression of the evaluative priming effect found here for self-generated actions.

Alternatively, response errors may be seen as a special instance of conflict, occurring between the actual and the post-correcting response (Botvinick, Braver, Barch, Carter, \& Cohen, 2001). In a recent study based on a similar evaluative priming paradigm, conflict produced by incongruent Stroop stimuli was shown to be more negative compared to nonconflict (i.e., congruent) Stroop stimuli (Dreisbach \& Fischer, 2012). Hence, it is possible that response errors evoke negative reactions because they involve conflict. However, more 
research is needed before it can be decided whether response errors and incongruent Stroop stimuli are evaluated as negative based on similar or different processes.

In our study, correct inhibitions (i.e., not responding to noGo stimuli) did not produce any evaluative priming effect. Schacht, Nigbur and Sommer (2009) previously found that nogo stimuli (presumably goal obstructive events) were not associated with physiological reactions corresponding to the detection of valence-specific events. Moreover, the lack of evaluative priming for correct inhibitions (or rejections) could be explained by the fact that when participants were able to withhold responding on nogo stimuli, the presentation of the subsequent target word occurred at least $1800 \mathrm{~ms}$ later $(1500 \mathrm{~ms}$ duration of the nogo stimulus plus the length of the SOA). These specific parameters were required to collect response errors on the same nogo stimuli. However, this interval was probably too long to enable an evaluative priming effect for correct inhibitions to take place.

Likewise, no clear evaluative priming effect was found following slow hits in our study. This might be due to the fact that the valence of slow hits (unlike either fast hits or response errors) was somehow equivocal. It is important to realize that the go-nogo task involved both goals regarding the nature of the responses (i.e., whether to press) and the speed of the responses (i.e., to respond quickly). Whereas a slow hit meets the first goal, it does not meet the second goal. Note that the goal to respond quickly was not only stated in the instructions but also implied by the feedback they received during the task. The absence of evaluative priming for slow hits might thus have reflected the ambivalence that participants experienced toward these stimuli.

For FAs, we not only observed evaluative priming but also post-error slowing (Laming, 1979; Rabbitt, 1966) as indicated by slower evaluative categorizations following FAs than hits (either fast or slow). However, evaluative priming was still highly significant when controlling for this general post-error slowing effect (by including it as a covariate in the 
statistical analyses), suggesting that these two effects (priming and slowing) may reflect different processes during action monitoring. Whereas post-error slowing likely deals with enhanced cognitive or attention control aimed at preventing errors to repeat over time (Botvinick et al., 2001; Notebaert et al., 2009), evaluative priming seems to reflect the online and internal tagging of a specific affective value (negative vs. positive) to a particular action (incorrect vs. correct). The notion that post-error slowing and evaluative priming presumably reflect different components of action-monitoring (e.g. enhanced cognitive or attentional control and genuine affective evaluation, respectively) is also supported indirectly by the differential modulatory effect of trait anxiety on these two components. Whereas the size of the evaluative priming effect did reliably correlate (negatively) with levels of trait anxiety ( $r$ $=-.28, p<.05)$, no such relationship was found between anxiety and post-error slowing $(r=-$ $.004, p=.98)$ even though evaluative priming and post-error slowing were clearly related to one another $(r=.42, p=.001)$. This latter relationship between evaluative priming and posterror slowing further suggests that the emotional tagging of the action may be boosted if more efforts are exerted to prevent errors to reoccur, consistent with recent theoretical accounts (see Verguts, Notebaert, Kunde, \& Wuhr, 2011). More generally, our new results are compatible with recent neuroscience findings showing that not only cognitive control systems are involved in action monitoring (and they likely include regions of the dorsal ACC besides deeper dopaminergic midbrain structures; see Klein et al., 2007), but also emotion control systems (including the amygdala) play an important role in this process, at a similar early latency following action execution (see Pourtois et al., 2010).

\section{Functional properties of automatic evaluation of actions}

Another important new result of our study concerns the actual time-course of the actionword evaluative priming effect. It is well established that especially at short intervals (SOA) between the prime and the target, a substantial priming effect is observed (Fazio et al., 1986; 
Hermans, De Houwer, \& Eelen, 2001). Here we found that the putative affective value of action influenced the subsequent evaluative categorization process only if the SOA was either $300 \mathrm{~ms}$ or $600 \mathrm{~ms}$. However, we did not find a similar evaluative priming effect with an SOA of $1000 \mathrm{~ms}$, whereas previous studies with word primes failed to find an effect with SOAs longer than $300 \mathrm{~ms}$. This discrepancy might be due to the task-relevance of the (action) prime in our experiments. More specifically, whereas the action primes in our experiments were self-generated and informative regarding performance on the Go/NoGo task, the prime words in previous studies were provided by the experimenter and essentially irrelevant for any task.

Finally, we observed that the evaluative priming effect was clearly related to the level of trait anxiety of our participants, as the evaluative priming effect became smaller with increasing levels of trait anxiety. This finding is in accordance with results from evaluative priming studies using external stimuli as primes that already reported blunted priming effects in high anxious participants (Berner \& Maier, 2004; Glaser \& Banaji, 1999; Maier, Berner, \& Pekrun, 2003). It is also consistent with previous action-monitoring studies which have shown that high trait anxious participants usually exhibit action monitoring deficits, as indicated by impairments to decode or read out the actual value of their actions using internal monitoring processes and swiftly relate it to (positive or negative) external performance feedback information presented in the environment (Aarts \& Pourtois, under revision; Hajcak et al., 2003).Together, these results suggest that in high trait anxious individuals, the rapid attribution process linking a specific value (either positive or negative) to an action (either correct or not) may somewhat be impaired, such that their online and internal action monitoring processes can in turn only weakly prime the immediately following evaluative categorization process. Alternatively, high anxious participants may show less priming than low anxious participants if they have a bias to focus exaggeratedly their attention on internal representations (Eysenck, 1992; Muris, Roelofs, Rassin, Franken, \& Mayer, 2005). However, 
this alternative account appears unlikely because high anxious participants were as fast (nonsignificant action type $\mathrm{x}$ word type $\mathrm{x}$ anxiety level interaction: $F(2,148)=1.14, p>.10)$ and accurate (non-significant action type $\mathrm{x}$ word type $\mathrm{x}$ anxiety level interaction: $F<1$ ) as low anxious participants in orienting towards the target word following the action. Likewise, high and low anxious participants emitted a similar number of FAs, $t(74)=-1.36, p>.10$, nor did they differ in response speed, $t<1$, during the speeded Go/noGo task. Hence, the present results also suggest that our new action-word evaluative priming paradigm may be suited to reveal specific impairments in action-monitoring processes, such as observed in psychopathological conditions or in individuals with certain personality traits (e.g., enhanced levels of trait anxiety).

It is important to note that in our analyses, we related trait anxiety to a compound RT measure of evaluative priming that was the average of the absolute priming effects for FAs and Fast Hits. We used this score because of evidence showing that modulatory effects of trait anxiety on (early) action monitoring processes are mostly generic (see Aarts \& Pourtois, 2010, 2012), and should thus apply to Fast Hits and FAs equally. When looking at each action type separately, we found a marginally significant correlation with anxiety following errors, $\mathrm{r}=-.24, \mathrm{p}=.07$, but a non-significant relationship following Fast Hits, $\mathrm{r}=-.08, \mathrm{p}>$ .10. However, little can be concluded from these additional analyses because the lack of significance could be due to a lack of statistical power and because the two correlation coefficients did not differ significantly from each other, $z=-.86, p>.10$

\section{Conclusions}

The results of this study show, for the first time, that the valence of simple self-generated actions are swiftly appraised. Unwanted FAs made during a simple Go/noGo task are actually perceived as more negative events compared to Fast Hits, while the latter events are perceived as more positive than the former outcomes. This affective appraisal of the action is 
in all likelihood based on the actual perceived goal conduciveness of the action, as achieved through a rapid and efficient internal action monitoring process. If a target word is presented within $600 \mathrm{~ms}$ following one of these two actions and shares the same intrinsic valence as the goal conducive or obstructive action, then participants are quicker and better at categorizing this word as either positive or negative, revealing a genuine action-word evaluative priming effect. No such effect is seen if 1000 ms elapses between the action and the onset of the target word, suggesting that this effect is short-lived and automatic. Finally, this effect is blunted in participants showing enhanced levels of trait anxiety, suggesting that (i) it is most likely the affective value of the action used as prime which is driving this strong evaluative priming effect; (ii) these participants have action monitoring difficulties in linking specific affective values (either positive or negative) to their self-generated actions (either correct or incorrect).

\section{Acknowledgements}

GP is funded by the European Research Council (Starting Grant \#200758) and Ghent University (BOF Grant \#05Z01708). JDH is supported by Methusalem Grant BOF09/01M00209 of Ghent University. We thank the three reviewers for their comments.

\section{References}

Aarts, K., \& Pourtois, G. (2010). Anxiety not only increases, but also alters early errormonitoring functions. Cognitive, Affective, \& Behavioral Neuroscience, 10(4), 479492.

Aarts, K., \& Pourtois, G. (2012). Anxiety disrupts selectively the evaluative component of performance monitoring: An ERP study. Neuropsychologia.

Beckers, T., De Houwer, J., \& Eelen, P. (2002). Automatic integration of non-perceptual action effect features: the case of the associative affective Simon effect. Psychological Research, 66(3), 166-173.

Berner, M. P., \& Maier, M. A. (2004). The direction of affective priming as a function of trait anxiety when naming target words with regular and irregular pronunciation. Experimental Psychology, 51(3), 180-190.

Botvinick, M. M., Braver, T. S., Barch, D. M., Carter, C. S., \& Cohen, J. D. (2001). Conflict monitoring and cognitive control. Psychological Review, 108(3), 624-652.

Cacioppo, J. T., Priester, J. R., \& Berntson, G. G. (1993). Rudimentary determinants of attitudes: II. Arm flexion and extension have differential effects on attitudes. Journal of Personality and Social Psychology, 65, 5-17. 
Chen, M., \& Bargh, J. A. (1999). Consequences of automatic evaluation: Immediate behavioral predispositions to approach or avoid the stimulus. Personality and Social Psychology Bulletin, 25, 215-224.

Danielmeier, C., \& Ullsperger, M. (2011). Post-error adjustments. Frontiers in Psychology, 2, 233.

De Bruijn, E. R. A., de Lange, F. P., von Cramon, D. Y., \& Ullsperger, M. (2009). When errors are rewarding. Journal of Neuroscience, 29(39), 12183-12186.

De Houwer, J., Hermans, D., \& Eelen, P. (1998). Affective and identity priming with episodically associated stimuli. Cognition and Emotion, 12, 145-169.

De Houwer, J., Teige-Mocigemba, S., Spruyt, A., \& Moors, A. (2009). Implicit measures: A normative analysis and review. Psychological Bulletin, 135, 347-368.

Defares, P. B., van der Ploeg, H. M., \& Spielberger, C. D. (1979). Zelf-Beoordelings Vragenlijst. Lisse: Swets and Zeitlinger.

Dreisbach, G., \& Fischer, R. (2012). Conflicts as aversive signals. Brain and Cognition, 78(2), 94-98.

Eysenck, M. W. (1992). Anxiety: The cognitive perspective. Hove: Lawrence Erlbaum Associates Ltd.

Fazio, R. H. (2001). On the automatic activation of associated evaluations: An overview. Cognition and Emotion, 15, 115-141.

Fazio, R. H., Sanbonmatsu, D. M., Powell, M. C., \& Kardes, F. R. (1986). On the automatic activation of attitudes. Journal of Personality and Social Psychology, 50, 229-238.

Fernandez-Duque, D., Baird, J. A., \& Posner, M. I. (2000). Executive attention and metacognitive regulation. Consciousness and Cognition, 9, 288-307.

Frijda, N. H. (1987). Emotion, cognitive structure, and action tendency. Cognition \& Emotion, 1(2), 115-143.

Giner-Sorolla, R., Garcia, M. T., \& Bargh, J. A. (1999). The automatic evaluation of pictures. Social Cognition, 17, 76-96.

Glaser, J., \& Banaji, M. R. (1999). When fair is foul and foul is fair: Reverse priming in automatic evaluation. Journal of Personality and Social Psychology, 77, 669-687.

Hajcak, G., \& Foti, D. (2008). Errors are aversive: Defensive motivation and the error-related negativity. Psychological Science, 19(2), 103-108.

Hajcak, G., McDonald, N., \& Simons, R. F. (2003a). Anxiety and error-related brain activity. Biological Psychology, 64(1-2), 77-90.

Hajcak, G., McDonald, N., \& Simons, R. F. (2003b). To err is autonomic: Error-related brain potentials, ANS activity, and post-error compensatory behavior. Psychophysiology, 40(6), 895-903.

Hajcak, G., McDonald, N., \& Simons, R. F. (2004). Error-related psychophysiology and negative affect. Brain and Cognition, 56(2), 189-197.

Hermans, D., Baeyens, F., \& Eelen, P. (1998). Odours as affective-processing context for word evaluation: A case of cross-modal affective priming. Cognition and Emotion, $12,601-613$.

Hermans, D., \& De Houwer, J. (1994). Affective and subjective familiarity ratings of 740 Dutch words. Psychologica Belgica, 34, 115-139.

Hermans, D., De Houwer, J., \& Eelen, P. (1994). The affective priming effect: Automatic activation of evaluative information in memory. Cognition and Emotion, 8(6), 515533.

Hermans, D., De Houwer, J., \& Eelen, P. (2001). A time course analysis of the affective priming effect. Cognition and Emotion, 15(2), 143-165.

Klein, T. A., Endrass, T., Kathmann, N., Neumann, J., von Cramon, D. Y., \& Ullsperger, M. (2007). Neural correlates of error awareness. Neuroimage, 34(4), 1774-1781. 
Laming, D. (1979). Autocorrelation of choice-reaction times. Acta Psychologica, 43(5), 381412.

Luu, P., Collins, P., \& Tucker, D. M. (2000). Mood, personality, and self-monitoring: Negative affect and emotionality in relation to frontal lobe mechanisms of error monitoring. Journal of Experimental Psychology, 129(1), 43-60.

Maier, M. A., Berner, M. P., \& Pekrun, R. (2003). Directionality of affective priming: Effects of trait anxiety and activation level. Experimental Psychology, 50, 116-123.

Moors, A., \& De Houwer, J. (2001). Automatic appraisal of motivational valence: Motivational affective priming and Simon effects. Cognition and Emotion, 15(6), 749-766.

Muris, P., Roelofs, J., Rassin, E., Franken, I., \& Mayer, B. (2005). Mediating effects of rumination and worry on the links between neuroticism, anxiety and depression. Personality and Individual Differences, 39, 1105-1111.

Neumann, R., Förster, J., \& Strack, F. (2003). Motor compatibility: The bidirectional link

between behavior and evaluation. In J. Musch \& K. C. Klauer (Eds.), The psychology of evaluation (pp. 371-392). Mahwah, NJ: Lawrence Erlbaum Associates, Inc.

Notebaert, W., Houtman, F., Van Opstal, F., Gevers, W., Fias, W., \& Verguts, T. (2009). Post-error slowing: An orienting account. Cognition, 111(2), 275-279.

Rabbitt, P. M. (1966). Errors and error correction in choice-response tasks. Journal of Experimental Psychology, 71(2), 264-272.

Reber, R., Haerter, A., \& Sollberger, B. (1999). Unbewusstes affektives Priming: Zwei neue experimentelle paradigmen. Paper presented at the 7. Tagung der Fachgruppe Sozialpsychologie.

Schacht, A., Nigbur, R., \& Sommer, W. (2009). Emotions in Go/NoGo conflicts. Psychological Research, 73(6), 843-856.

Scherer, K. R. (1984). On the nature and the function of emotions: A component process approach. In K. R. Scherer \& P. Ekman (Eds.), Approaches to emotion (pp. 293-317). Hillsdale, NJ: Erlbaum.

Scherer, K. R. (1988). Criteria for emotion-antecedent appraisals: A review. In G. H. Hamilton, G. H. Bower \& N. H. Frijda (Eds.), Cognitive perspectives on emotion and motivation (pp. 89-126). Dordrecht: Kluwer.

Spielberger, C. D. (1983). Manual for the State-Trait Anxiety Inventory. Palo Alto, CA: Consulting Psychologists Press.

Vansteenwegen, D., Crombez, G., Baeyens, F., \& Eelen, P. (1998). Extinction in fear conditioning: Effects on startle modulation and evaluative self-reports. Psychophysiology, 35(6), 729-736.

Verguts, T., Notebaert, W., Kunde, W., \& Wuhr, P. (2011). Post-conflict slowing: cognitive adaptation after conflict processing. Psychonomic Bulletin \& Review, 18(1), 76-82.

Vocat, R., Pourtois, G., \& Vuilleumier, P. (2008). Unavoidable errors: A spatio-temporal analysis of time-course and neural sources of evoked potentials associated with error processing in a speeded task. Neuropsychologia, 46(10), 2545-2555.

Winkielman, P., Schwarz, N., Fazendeiro, T. A., \& Reber, R. (2003). The hedonic marking of processing fluency: Implications for evaluative judgment. In J. Musch \& K. C. Klauer (Eds.), The psychology of evaluation (pp. 189-217). Mahwah, NJ: Lawrence Erlbaum. 


\section{Figures caption}

Figure 1. Stimuli and task. On each trial, a black arrow was presented (either upright or inverted). After a variable interval of 1000-2000 ms, the black arrow turned either green or turquoise. Participants had to respond by pressing a button of the response box as fast as possible with their non-dominant hand only when the arrow became green and kept its initial orientation (A), but not otherwise (B). This first action was then followed (300 ms in Experiments 1-2, $600 \mathrm{~ms}$ in Experiment 3 or $1000 \mathrm{~ms}$ in Experiment 4) by either a positive or negative target word that had to be classified as either positive or negative by pressing one of two predefined keys on the response box using their dominant hand. After this emotional word categorization, participants received a general feedback about their performance for the two tasks for this specific trial. Accuracy regarding the speed for correct responses (on Go trials) was determined based on a stringent procedure and response deadline (see Methods).

Figure 2. Mean RTs (+ 1 standard error of the mean (SEM) for bars) for correct evaluative categorizations as a function of prime type (FA, Fast Hit or Slow Hit) and word type (Negative or Positive Words) in (A) Experiment $1(\mathrm{SOA}=300 \mathrm{~ms}),(\mathrm{B})$ Experiment 2 (SOA $=300 \mathrm{~ms}),(\mathrm{C})$ Experiment $3(\mathrm{SOA}=600 \mathrm{~ms})$ and $(\mathrm{D})$ Experiment $4(\mathrm{SOA}=1000 \mathrm{~ms}) .{ }^{*} \mathrm{p}<$ .05 .

Figure 3. Mean accuracy (+ 1 standard error of the mean (SEM) for bars) for evaluative categorization as a function of prime type (FA, Fast Hit or Slow Hit) and word type (Negative or Positive Words) in (A) Experiment 1 (SOA = 300ms), (B) Experiment 2 (SOA $=300 \mathrm{~ms}),(\mathrm{C})$ Experiment $3(\mathrm{SOA}=600 \mathrm{~ms})$ and $(\mathrm{D})$ Experiment $4(\mathrm{SOA}=1000 \mathrm{~ms}) .{ }^{*} \mathrm{p}<$ .05 . 
Figure 4. Significant negative correlation between evaluative priming effect [measured as the difference in RT latency between incongruent trials (i.e., FAs-Positive and Fast HitsNegative) and congruent trials (i.e., FAs-Negative and Fast Hits-Positive)] and levels of trait anxiety (measured using a standard questionnaire, see Methods). This correlation was calculated for participants of Experiments 1-3 together where a significant evaluative priming effect was evidenced. 